\title{
Volume sculpting based on geometric tools
}

\author{
Rafael Huff, Roberto Silveira da Rosa Jr., Luciana Nedel, Carla Maria Dal Sasso Freitas* \\ Institute of Informatics, Federal University of Rio Grande do Sul, \\ 91501-970, Porto Alegre, RS, Brazil
}

Received: October 22, 2008; Accepted: June 23, 2009

\begin{abstract}
The analysis of volumetric datasets is the main concern in many areas ranging from geophysics to biomedical sciences. The direct visualization of these data plays an important role in this scenario, and in spite of developments in volume visualization techniques, interacting with large datasets still demands research efforts due to perceptual and performance issues. There is a need of interactive sculpting tools which can provide an intuitive way to examine and explore inner parts of the datasets, as well as to fill missing data for specific purposes. In this paper we report the development of interactive, intuitive and easy-to-use sculpting tools, which specify regions within the volume to be discarded from rendering, thus allowing inspection of the volume interior, and to be filled with material to build virtual structures in the volume. Interactive rates for these sculpting tools were obtained by running special fragment programs on the graphics hardware. The tools were implemented using two interaction metaphors (virtual pointer and virtual hand) and following different approaches in terms of devices and single versus two-handed interaction. We report the evaluation of these approaches in detail and concluded that the use of two different devices together presents a better performance and are preferred by users. Moreover, the use of virtual hand interaction provided better results than using the virtual pointer during the tests.
\end{abstract}

Keywords: volume sculpting, virtual pointer, virtual hand, volumetric data, volume rendering.

\section{Introduction}

Several applications involve the generation, exploration and analysis of volumetric datasets. For years, the requirements of medical applications have pushed the stateof-the-art in visualization and interaction techniques, mainly driven by accuracy and computational time constraints.

Direct visualization of volume data, or volume rendering, has been playing an important role in this scenario allowing to obtain helpful images through the use of a variety of associated techniques: from colors and transparency ${ }^{29,19,18}$ to enhance or fade away portions of the dataset to techniques used by scientific illustrators to improve perception of some structures $^{10,3}$; from geometric clipping planes to allow the observation of arbitrary slices of the dataset ${ }^{22,30}$ to volume deformation techniques ${ }^{23,7}$ to provide ways of browsing the volume.

In direct volume rendering, transfer functions ${ }^{29}$ provide a mapping between data values and image visual properties. However, although there have been a considerable research effort on developing these techniques, the generation of informative renderings from a dataset is still challenging. The specification of efficient transfer functions ${ }^{11}$ to segment some datasets is sometimes impracticable, even with recent frameworks ${ }^{8,41}$, requiring non-automatic segmentation tools based on interactive manipulation of the volume data. For example, it is difficult to isolate some inner structures of brain CT scans due to the resemblance of their density values with others in the volume, and frequently an expert is required to manually separate these structures using geometric tools.
In order to assist users to perform this task, volume clipping and deformation tools have been developed ${ }^{30,38,23,4}$. However, especially in clipping tools, most of the research is dedicated to improve performance in order to guarantee interactive rates, and few of them are concerned with the interactivity itself. Therefore the objective of our work is to develop intuitive, easy-to-use and efficient clipping tools (Figure 1), generic and simple enough to be used in volumetric datasets from different fields. In order to guarantee that, we performed many test with users to evaluate the interaction with the tools.

The operations performed to clip volumes, removing material resembling a manual modeling task, have been referred as volume sculpting ${ }^{37}$. More recently, Islam et al. ${ }^{17}$ coined the expression volume splitting as a general form of referring to both deformation and clipping operations. They base their work on constructive volume geometry, which is a nice concept that provides the basis for an important operation in many applications, the insertion of material into parts of the volume. For example, in the volume shown in Figure 2a part of the jaw is missing due to trauma, and the insertion of material in those voxels can be used as an initial step for designing a prosthesis (Figure $2 b$ ).

Traditional volume sculpting is basically a selection task, where the voxels inside a volume are first selected (using some tool like the carving tools in Wang and Kaufman ${ }^{37}$ ) and then deleted. For adding a structure to empty voxels, in this modeling process, the same actions could be done: selecting voxels to fill them with a specified material. 

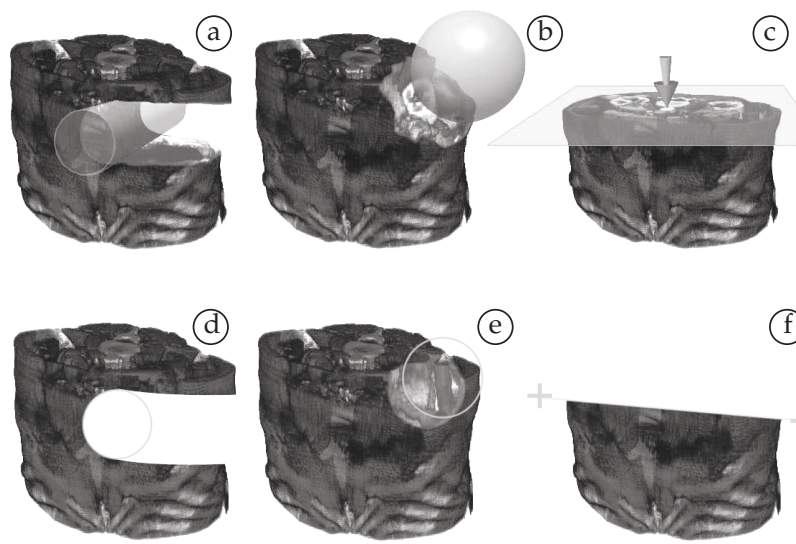

(e)

(f)

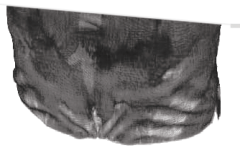

Figure 1. Volumetric Sculpting Tools: a) 3D Eraser, b) 3D Digger, c) 3D Clipper, d) 2D Eraser, e) 2D Digger and f) 2D Clipper.
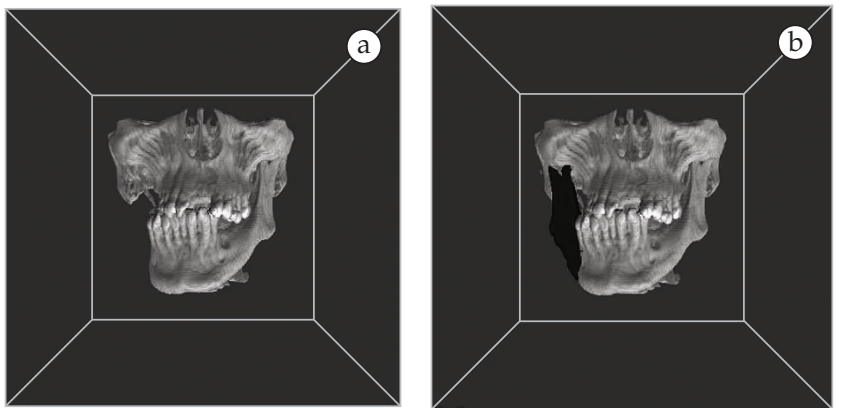

Figure 2. a) Part of the jaw (left side) is missing due to trauma; and b) Voxels filled with material to model a prosthesis.

The selection of these voxels can be made using many different techniques represented by two basic metaphors ${ }^{33}$ : virtual pointer and virtual hand. In the virtual pointer metaphor, the user points at the object of interest using some cursor even if he is far away from the object. Techniques based on the virtual pointer metaphor are usually easy to learn and use because they are based on 2D interaction, i.e., in tasks with two degrees-of-freedom (DOFs), and nearly everybody is familiar with 2D input devices and applications. However, in 3D interaction techniques based on the virtual hand metaphor, the continuity between the real and virtual world is enhanced, because the cursor has six DOFs and allows the user to "touch" and manipulate virtually the object of interest.

In an earlier paper ${ }^{16}$ we described three sculpting tools following a 3D interaction paradigm - the virtual hand interaction technique - to allow real-time volume sculpting in an efficient and comfortable way while using commodity PC workstations. Our 3D tools are called: 3D eraser, 3D digger and $3 D$ clipper (Figures 1a-c). Real-time response rates have been achieved by running the sculpting tools and volume rendering on GPU. In that work we were interested in measuring user's performance using different devices (conventional and 3D mouse) as well as one and two hands.

The present paper reports a large extension of our previous work comprising several aspects. We implemented the three sculpting tools following also a $2 \mathrm{D}$ interaction paradigm, using the virtual pointer interaction technique (Figures 1d-f). The efficiency of the interaction was measured regarding different ways of controlling the tools. Like in the previous work, we implemented the tools controlled by a conventional 2D mouse as well as a 3D mouse. As a result of experiments with users, we also designed and implemented two additional sculpting operators, for inserting material into the volume, thus fulfilling the lack we found in the current scenario regarding volume manipulation. These operators are mirror and filler, which we classify as filling operators. They were assessed by exploring alternatives in a usage scenario.

The remainder of the paper is structured as follows. In the next section, we review some relevant previous work. We then present the sculpting tools (Section 3) including details of their implementation (Section 4). The use of the tools by experimental users is reported and discussed (Section 5). The filling operators are described and their evaluation is reported in Sections 6 and 7, respectively). Conclusions and future work are drawn in the last section.

\section{Related Work}

Although volumetric visualization techniques have been proposed since the 80 's, only in the last years the advances in commodity graphics hardware allowed texture-based volume rendering to achieve satisfying image quality with better performance than software-based methods ${ }^{34,19}$.

Interacting with volumes to expose inner structures through clipping tools started with the use of clipping planes $^{22,14,28}$. Wang and Kaufman ${ }^{37}$ pioneered the use of carving and sawing tools, with voxelized geometries as tools and explicit set operators between volume data and the tools. Along the years, different clipping approaches have been considered in scientific visualization ${ }^{30,39}$.

Van Gelder and $\mathrm{Kim}^{14}$ used clipping planes to specify the boundaries of the clipping region in texture-based volume rendering while Westermann and $\operatorname{Ertl}^{40}$ introduced the concept of clipping geometries by means of stencil buffer operations. Later on, Pfister et al. ${ }^{28}$ proposed the VolumePro hardware architecture with cropping and cutting planes features for clipping the volume.

Weiskopf et al. ${ }^{38}$ proposed clipping tests using perfragment operations on the graphics hardware to maintain high rendering speed. They propose a depth-based clipping technique that analyzes the depth structure of the clipping geometry to decide which parts of the volume have to be clipped (a similar approach is used by Diepstraten et al. ${ }^{9}$ ). They also propose a clipping tool that uses a voxelized clipping object to identify clipped regions. In this tool, a texture buffer stores visibility information, and it is used as a mask that indicates if each voxel will contribute to the final image. This clipping texture is obtained by the voxelized geometry of clipping tools on the CPU. Scale, rotation and translation operations of the clipping object are allowed by adapting the texture coordinates of the clipping texture. The "voxelized 
clipping object" paradigm is also exploited in our work but the geometries of our tools are voxelized on the GPU.

Volume deformation tools, as proposed by McGuffin et al. $^{23}$, have the advantage of showing inner structures surrounded by its context. However, as presented in that work, they do not serve to the purpose of actually segmenting parts of interest in the volume since they are devoted mainly to browsing purposes. They provide a way to explore semantic layers in the dataset (i.e. previously segmented data), by spreading voxels in a given direction. In the present work, regarding clipping, we are concerned with the actual isolation of voxels, removing from the view those that are not of interest.

VolumeShop, proposed by Bruckner and Gröller ${ }^{4}$, is a volume illustration system based on sculpting tools: cutaway and ghost tools use opacity assignments to remove occluding regions thus providing visual inspection of inner features. This is somehow similar to our approach, the difference relying on the way we select the regions to be eliminated from (or added to) the volume. They cast a ray from a point clicked on a screen into the volume. At the first voxel crossed by this ray, a volumetric brush centered on the voxel can either add to or erase details from the selection volume for every voxel within the brush bounding box.

Recently, Chen et al. ${ }^{6}$ also presented a framework of interactive GPU-based tools for real-time volume manipulation and segmentation. These tools were developed using a pointbased strategy for sculpting volumes and removing occluding materials. Some operations of these tools were inspired by medical procedures like drilling, peeling and lasering, and others provided basic operations like cutting and pasting. They also implemented a system for region-growing segmentation and multiple seeds planting with sketches.

The research briefly reviewed above proposed new techniques to improve the performance of sculpting tools, while guaranteeing interactive frame rates. However, the authors do not exploit the advantages of different interaction metaphors and devices, although sculpting applications for modeling purposes have used different devices, such as conventional 2D mouse ${ }^{37}, 3 \mathrm{D}$ mouse ${ }^{13}$, and even both devices at the same time ${ }^{27}$.

In our previous work $^{16}$, we developed three sculpting tools (3D eraser, $3 D$ digger and $3 D$ clipper) following the virtual hand interaction technique, using different devices (conventional and 3D mouse) as well as one and two hands. Experiments showed that the conventional 2D mouse yielded better performance in time completion for the eraser and digger tools, but error rate was lower when using the 3D mouse for controlling the sculpting tool in two-handed interaction. User's preference obtained through a questionnaire showed that subjects preferred to use the 2D mouse to control the sculpting tool and the 3D mouse to control the volume.

Even though interaction techniques could be classified into just two basic metaphors - virtual pointer and virtual hand - several different techniques should be considered to implement these metaphors. For the virtual pointer meta- phor, techniques can differ from each other in the shape of the pointer, the definition of its direction and the methods of disambiguating the object the user wants to select. The most common example is the ray-casting technique ${ }^{24}$ and its variations: ray-casting with fishing reel ${ }^{1}$, spotlight ${ }^{25}$, aperture selection $^{12}$, and image plane ${ }^{31}$. Regarding the virtual hand based techniques, the choice of input devices and mappings between the position and orientation of real and virtual hands are the differentiation. The classic virtual hand was discussed by many authors ${ }^{33,36,26}$ and some extensions have been presented ${ }^{32,1,24}$.

Moreover, two-handed input has often been viewed as a technique to improve the efficiency of human-computer interaction, by enabling the user to perform two sub-tasks in parallel ${ }^{5}$, helping users to better perceive a 3D environment because it takes advantage of people's innate ability of knowing precisely where their hands are relative to each other ${ }^{15,35}$. Leganchuck et al. ${ }^{20}$ also noticed that using both hands in an application brings cognitive and manual advantages and their work provided a complimentary perspective, exploring the potential benefits into everyday applications.

In the present work we exploit all these alternatives to enhance interactivity in volume sculpting while maintaining high-quality and real-time rendering by employing graphics hardware programming.

\section{Tools for Volume Sculpting}

Aiming at an intuitive interface for sculpting volumes, we designed a set of tools based on interaction techniques using both the virtual pointer and the virtual hand metaphors.

Since selection using virtual pointer is essentially a 2D operation ${ }^{2}$, we developed a set of 2D sculpting tools using the "painting" paradigm present in most ordinary imageediting applications, where the user controls virtual pens, brushes, and other free-hand artistic instruments to paint or erase on a virtual canvas. These tools are called 2D eraser, $2 D$ digger and 2D clipper (Figures 3a-c), and implement basic sculpting operations, which are used to perform complex cutting, clipping and cropping tasks. Even though the user is sculpting a $3 \mathrm{D}$ volume, using these $2 \mathrm{D}$ tools is like editing a common 2D image.

The three-dimensional versions of the same tools (3Deraser, $3 D$ digger and $3 D$ clipper, see Figures $3 d-f)$, reported in Huff et $a .^{16}$, use the virtual hand metaphor. In these versions, the virtual hand is represented as different cursors, depending on the tool in use, and their shapes mimic the operation of the 2D tools. The following sections describe the conceptual design of the $2 \mathrm{D}$ and $3 \mathrm{D}$ tools as well as their implementation.

\subsection{Eraser tools}

The 2D Eraser tool (Figure 1d) uses the Aperture Selection technique $^{12}$, a modification of the ray-casting technique. The cursor is a circle aligned with the view plane and the origin of the virtual pointer is set to the location of the user's point of view, as shown in Figure 3a. 


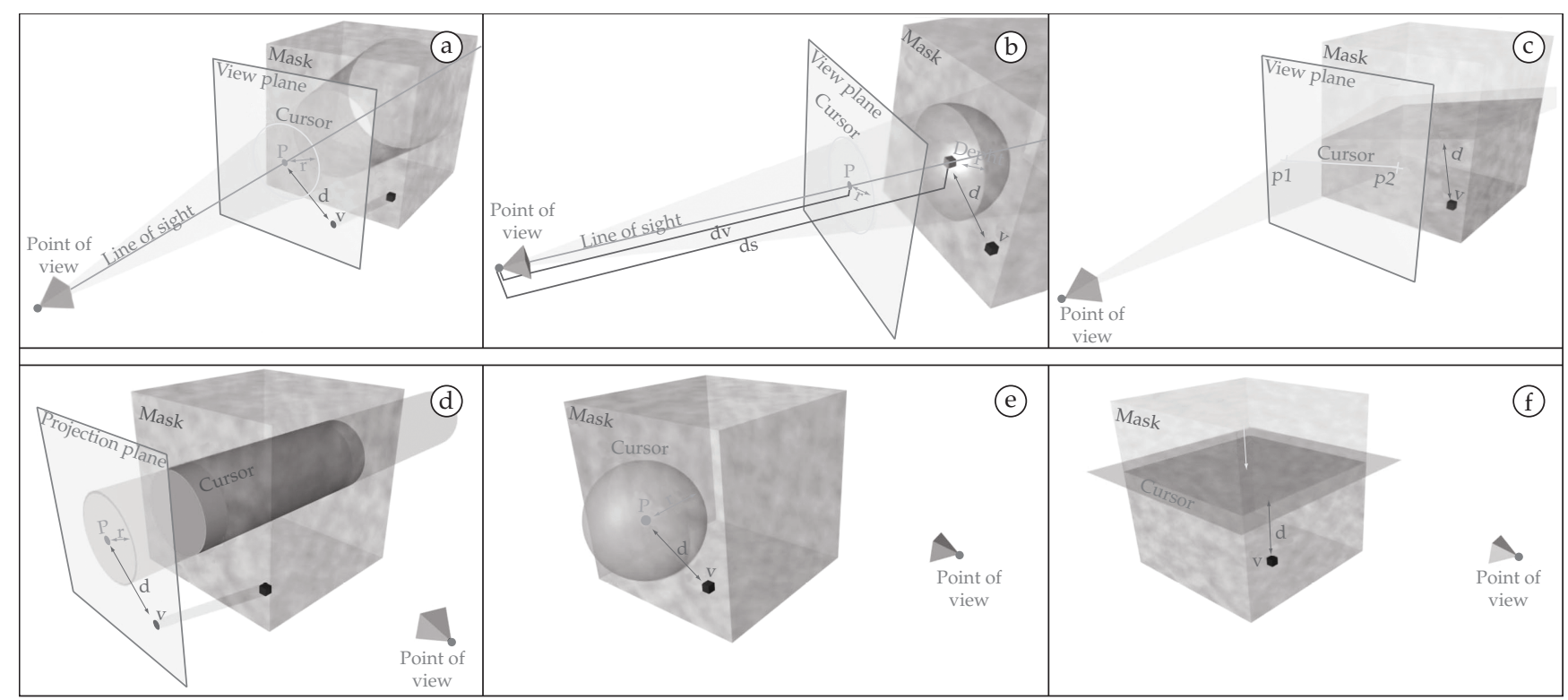

Figure 3. 2D Tools: a) Eraser, b) Digger, and c) Clipper. 3D Tools: d) Eraser, e) Digger, and f) Clipper.

The direction vector of this pointer, named line of sight, is the vector from the point of view to the center of the cursor. The size of the cursor $r$ is previously established by the user and the position $P$ is also specified by the user through the selection of a pixel on the screen. The size and 2D position of the cursor, together with its distance to the point of view, determine the selection volume.

When the 2D Eraser tool is applied to the volume, the position of each voxel is tested against the selection volume, and all voxels inside this volume are removed. The test is performed by (perspective) projecting the center of each voxel onto the view plane. If the 2D Euclidean distance $d$ of the projected voxel $V$ to the center of the cursor $P$ is smaller than the radius $r$ of the cursor, the voxel is inside the volume (Figure 3a).

Observing Figure 3a, it is possible to notice that the 2D Eraser Tool can be interpreted in 3D as a cone (due to the perspective projection during the volume visualization process) perpendicular to the view plane with the apex located at the user's point of view and the base at the infinity. The viewing angle is the opening angle of the cone, considering a cross section through the apex point and the center of the cursor.

Using an orthographic projection, the resulting shape is a cylinder, which makes more sense when the virtual hand metaphor is used for controlling the tool, since the cursor should be independent of viewing parameters. Detaching the cylinder from the point of view of the user provides the possibility of freely rotate and translating it. Our 3D Eraser tool (Figure 1a) was conceived in this way (Figure 3d).

The parameters used for testing a voxel against the volumetric region defined by the cylinder (3D Eraser tool) are similar to those employed for the 2D Eraser tool: a projection plane, a 2D point $P$ on this plane and the radius $r$ of the cylinder. It should be noticed that in the 3D Eraser, the cursor is independent of the viewing parameters, and thus the projection and the view plane are independent too, as can be observed in Figure 3d). When the cursor is rotated and translated, these transformations are applied to this projection plane. The selection of a voxel for elimination is done by first orthogonally projecting its center into the projection plane. For each projected center, a distance to $P$ is computed (called projected distance $d$ of a voxel). $d$ is a 2D Euclidean distance since it is calculated on the projection plane (Figure 3d). Following, every voxel with $d<r$ is removed.

\subsection{Digger tools}

Often, during volume sculpting, instead of erasing it all the way through with the Eraser tools, the need is digging a hole in the volume. Here, this is accomplished by the Digger tools.

The 2D Digger tool (Figure 1e) considers for removal the same voxels of the 2D Eraser tool, i.e., inside a circle, but only those within a given depth from the first point on the volume boundary along the line of sight (Figure $3 b$ ). The cursor of this tool is also a circle that can be translated (by the user) on the view plane, and its depth is calculated from the cursor radius $r$ (also defined by the user), the surface distance $d s$ and the view plane distance $d v$ to the point of view, as given by Equation 1 .

$$
\text { depth }=\frac{\mathrm{ds}}{\mathrm{dv}} * \mathrm{r}
$$

When the 2D Digger tool is applied, it is like an object hitting the surface of the volume and eliminating every voxel inside a region delimited by its depth. This is obtained by selecting the first voxel (on the surface of the volume) intercepted by the line of sight and calculating the 3D Euclidean distance $d$ from it to every other voxel $v$. All the voxels within a distance smaller than the depth of the tool are erased. The 
selection region associated to the $2 \mathrm{D}$ Digger tool can be interpreted as a semi-sphere with the center located on the surface of the volume. The size and position of this semi-sphere are constrained by viewing parameters: its size varies according to Equation 1, and the position is determined by the intersection between the line of sight and the volume surface.

As for the 3D Digger tool, the virtual hand cursor is actually a sphere detached from the view plane. This means that the center of the sphere does not have to be located on the volume surface anymore, and can be placed anywhere in the volume, being freely controlled by the user (Figure 1b). Figure 3 e shows how it was designed. The sphere has radius $r$ and center position $P$ both specified by the user, and the selection of a voxel for removal is done by first calculating the 3D Euclidean distance $d$ of the voxel's center to $P$. If this distance is smaller than $r$, the voxel is removed.

\subsection{Clipper tools}

Differently from the 2D Eraser and Digger tools, the cursor of the 2D Clipper tool (Figure 1f) is represented by a line segment on the view plane (Figure 3c), defined by two points selected by the user on the screen. This line divides the whole image that the user is observing, in order to delete part of the volume.

The 2D Clipper defines a convex region that lies inside the $3 \mathrm{D}$ original volume following a carving approach. This is accomplished by calculating a clipping plane through three points: the point of view and the two points specified by the user on the screen. The selection of a voxel for elimination is done by calculating the smallest distance of its center to that plane. This (signed) distance is calculated by a dot product between voxel coordinates and plane coefficients. The voxel is removed if the distance is negative. Even though a clipping plane and 3D distances are used, due to the perspective projection, the user visualizes it as a $2 \mathrm{D}$ operation.

As for the virtual hand metaphor (Figure 1c), instead of a line, the user manipulates a square (Figure $3 f$ ) that represents the cutting plane. The square can be freely translated and rotated, and, differently from the 2D Clipper tool, it is not attached to the user's point of view. When this cutting plane is applied for removing voxels, it slices the current volume and creates a shape with one additional face. This process can be repeated as long as needed, and is performed using the dot product, like for the 2D Clipper tool.

\section{Tools Implementation}

All the six sculpting tools presented in the previous section (2D and 3D Eraser, Digger and Clipper) were integrated in a texture-based volume rendering system based on the paradigm proposed by Weiskopf et al. ${ }^{38}$. Figure 4 shows the structure of the system using a clipping plane as an example of volume sculpting tool.

The first stage (initialization) is the processing of the volumetric dataset and its storage as a 3D texture in the GPU. During this process, if required, a pre-classification according

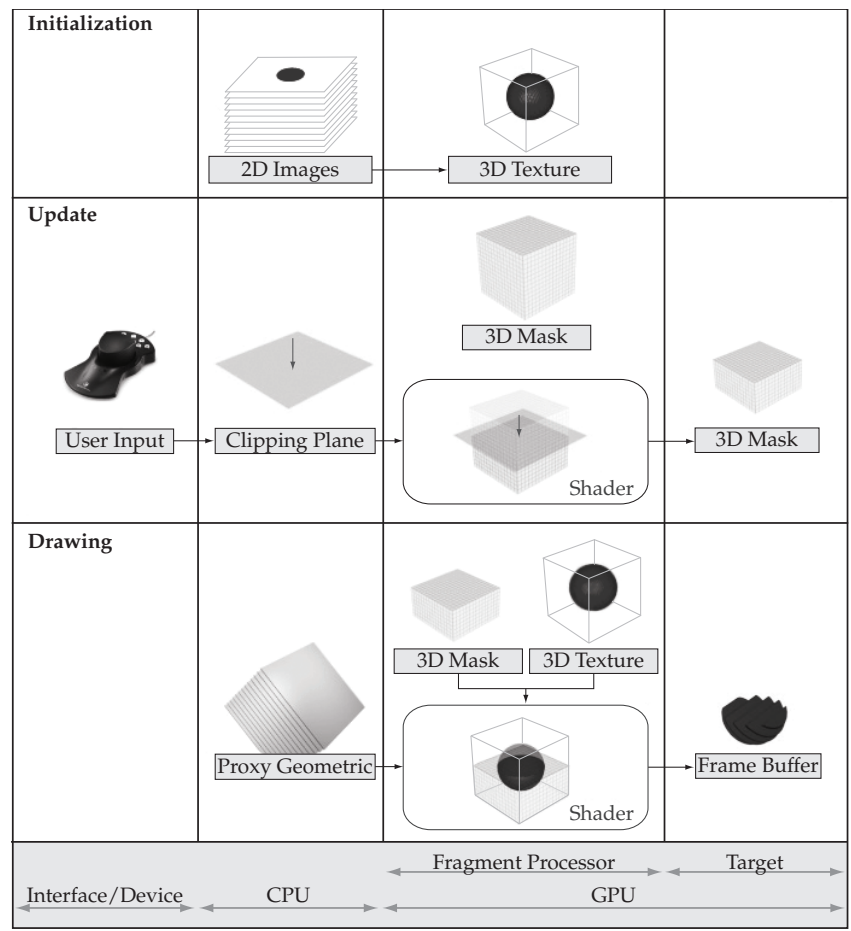

Figure 4. Clipping example: a volume containing a red sphere is clipped.

to a transfer function ${ }^{11}$ or calculations of scalar values and gradients for volume shading ${ }^{39}$ can be used.

The actual sculpting of the volume is accomplished through special fragment shaders that run on the GPU, and use the tool geometry to eliminate voxels. This is accomplished by the stages update and drawing.

With few parameters, the geometries of our tools are voxelized on the GPU by specialized fragment shaders, differently from the work developed by Weiskopf et al. ${ }^{38}$, where tools are voxelized on the CPU.

Updates are requested when the user interacts with the system, either changing viewing parameters or sculpting the volume. Viewing changes are issued at camera manipulation, but sculpting can be produced by any tool. Since the tools are voxelized on the GPU, the active clipping tool can be changed interactively, resulting in real time tools manipulation and volume sculpting operations. If a sculpting tool is applied to a volume, voxels that lie within the geometry associated to the tool are marked to be removed, resulting in a 3D mask. Values associated with marked voxels can be either binary ( 1 = visible; 0 = removed) or Euclidean distances to the closest point on the clipping object, in order to avoid jaggy artifacts. This is accomplished by fragment shaders.

The fragment shader that implements the eraser tools is called FSEraser, and receives as input the 3D mask (SculptMap), the projection plane, the 2D point $P$, and the tool radius $r$ (refer to Figures $3 \mathrm{a}, \mathrm{d}$ ). For each fragment in the proxy rectangle, the steps shown in Figure 5 are performed.

The digger tools (Figure 3b, e) are implemented by the same fragment shader called FSDigger shown in Figure 6. The inputs for the shader are the 3D mask SculptMap, the point $P$ and the cursor radius $r$. 


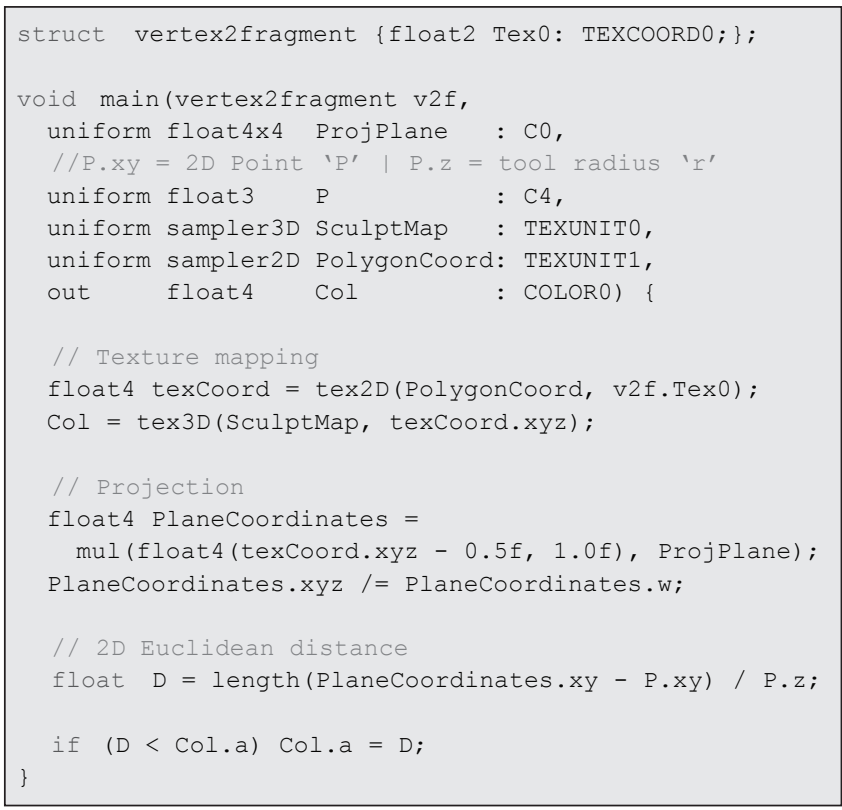

Figure 5. Cg code for FSEraser.

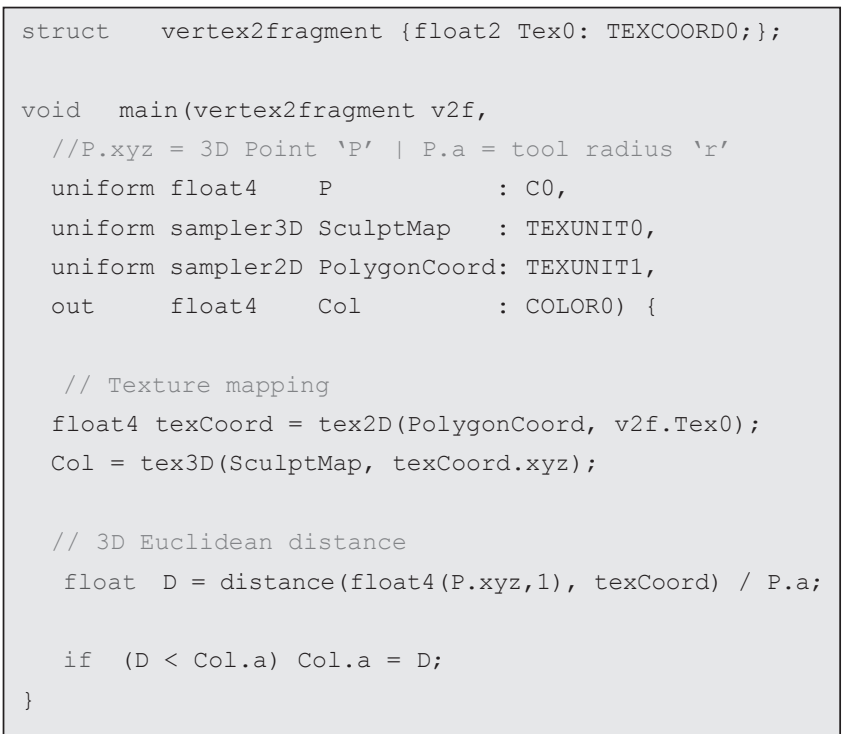

Figure 6. Cg code for FSDigger.

Finally, FSClipper is the fragment shader that implements the clipper tools (Figures 3c, f). As the other shaders, the SculptMap is a parameter for this one, and the cutting plane completes the arguments needed for rendering the result of the clipper tools. Cg code for FSClipper is shown in Figure 7.

The visualization of the dataset is achieved by sampling a 3D texture using a set of planes aligned with the viewing direction as proxy geometry (see drawing phase in Figure 4). During the rendering stage, these planes are drawn in sorted order. For the 3D texture to be sampled, we use the combination of two different $3 \mathrm{D}$ volumes, one with the original dataset, and a second one, the texture mask SculptMap, with visibility information. Voxels marked to be removed have their alpha values modified in the texture mask. The visualization is performed by combining the original dataset with the texture

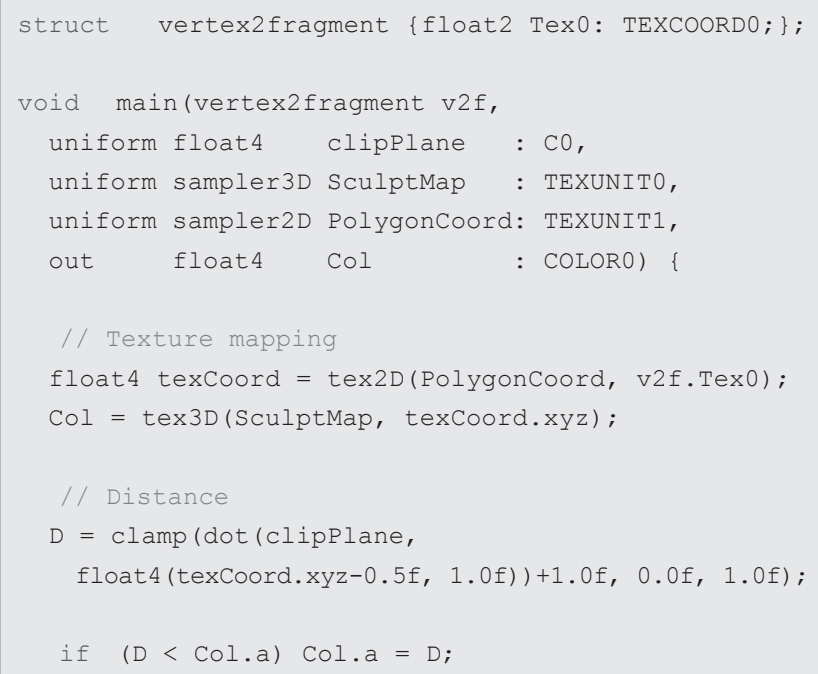

Figure 7. Cg code for FSClipper.

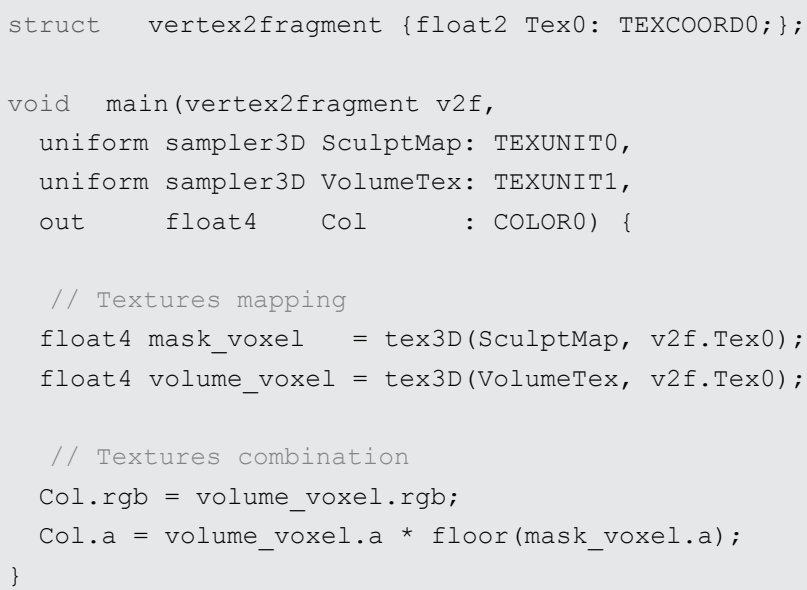

Figure 8. Cg code for FSCombiner.

mask, discarding marked voxels which should not contribute to the final image. The texture mask is frequently smaller than the original volume and a tri-linear interpolation of visibility values should also be considered during the combination process. In our system, the combining step is performed in a fragment shader, called FSCombiner (Figure 8).

\section{Testing the Volume Sculpting Tools}

In our work, we aimed at developing efficient, precise, intuitive and comfortable interactive tools. Based on these criteria of quality, we conducted two sets of tests with users. Since the tests were not planned for expert users from a specific field, we used generic volumes, which every user could understand and complete the task. For both tests, the experiments consisted of the elimination of red voxels from red-and-grey volumes (Figure 9). 


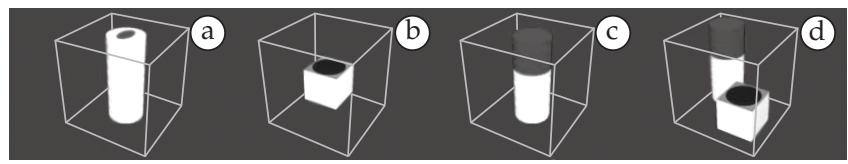

Figure 9. Experiment datasets: a) eraser evaluation volume, b) digger evaluation volume, c) clipper evaluation volume and d) training evaluation volume.

Considering the assumption that using 2D tools is as easy and natural as the editing of common 2D images with a mouse in a "painting" system, the first set of tests was designed to evaluate the preference and performance of users when manipulating and carving volumes with the 3D tools. In these tests, the user could choose between a regular mouse, a 3D mouse, or both concurrently to manipulate the volume and the tools. Section 5.1 presents details of the experiment reported in Huff et al. ${ }^{16}$.

The second set of tests intended to verify which interaction metaphor (virtual hand or virtual pointer) is the most appropriate for sculpting volumes. These tests actually compare user performance and preference between 2D and 3D sculpting tools. Details are presented in Section 5.2.

\subsection{Which is the best device to control $3 D$ tools?}

The main goal for using $3 \mathrm{D}$ tools is to enlarge the continuity between visualization and interaction. In the first set of tests, we adopted the virtual hand as the 3D interaction metaphor to control the tools, so the user is not forced to decompose a 3D task into a series of 2D or 1D tasks ${ }^{21}$.

We used a Magellan Space mouse (see Figure 10, upper left part), since it has 6 degrees of freedom (DOFs), and it is relatively simple, robust and easy to learn. However, for evaluation purposes, we also implemented the virtual hand metaphor controlled by a conventional mouse since it is the most commonly used input device.

In our testbed application, two different objects can be manipulated by the input devices: the tools and the volume of interest (VOI). The tools can be rotated around themselves and translated in $x, y$, and $z$ axis. The VOI can also be rotated around itself but cannot be translated. While the 3D mouse allows easy control of 3D rotations and translations on a virtual hand interaction, these transformations are not trivially executed with a conventional 2D mouse.

In order to compare both devices, we had to compensate the lack of DOFs of the 2D mouse. Translations along the $x$ and $y$ axis were normally performed by moving the $2 \mathrm{D}$ mouse, but translations along the missing $z$ dimension were performed by rolling the wheel of the common mouse (Figure 10). Rotations were performed by holding down a button and moving the mouse around in a dragging movement.

The user could select in the interface of the application which object (tools or VOI) he/she wanted to manipulate with each mouse (2D or 3D). The manipulation of both objects with the same device at the same time was not allowed. The

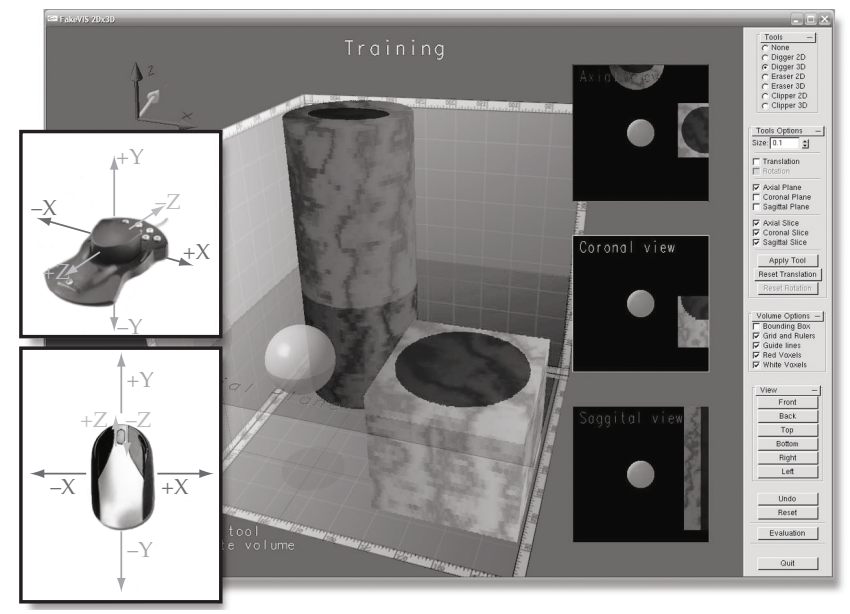

Figure 10. System interface and the interpretation of input devices operation to manipulate 3D tools.

user would have to switch between them explicitly. In order to manipulate both objects at the same time, two-handed interaction was enabled by using the 2D and 3D mouse at the same time. In this case, the user could choose which device would be associated with each object (tools and VOI). Due to implementation issues, two 3D mice or two 2D mice could not be used at the same time.

Our evaluation was based on three hypotheses, concerning to the combination of input devices used:

- H1: The manipulation of tools is faster using a conventional mouse.

- H2: The error rate decreases when the subjects adopt the $3 \mathrm{D}$ device to manipulate the tool.

- H3: The subjects will prefer two-handed input, using both devices simultaneously to manipulate volume and tools.

The first hypothesis rises from the thought that common users are used to the conventional mouse and a training stage with the 3D mouse would not provide them the same skills. The second hypothesis, on the other hand, is based on the statement that 3D devices are better suited for 3D interaction techniques ${ }^{21}$. Finally, the last hypothesis relies on the cognitive benefits that reduce the load of mentally composing and visualizing the tasks at an unnatural low level which is imposed by traditional single-handed techniques ${ }^{5}$.

\subsubsection{Scenario and subjects}

The experiment consisted of the elimination of red voxels in a red-and-grey volume. The testbed application takes as input different datasets, which are volumes composed by a set of images with red and grey pixels. We designed three different datasets, one for each tool, because of the geometry associated with each tool. A fourth volume was used to train the users before they start the evaluation process (Figure 9).

The user has to remove the maximum number as possible of red voxels from the volume, while avoiding the elimination of the grey ones as well. 
To test the three hypotheses we defined 12 user tasks consisting of a basic task performed with different combination of input devices. The combination of three sculpting tools (digger, eraser and clipper), two different objects (tools and volume) to be manipulated as well as two input devices (2D and 3D mouse) resulted in the set of 12 different tasks.

We have performed tests with 15 subjects (one professor and 14 students), most of them with a Computer Science major. Even though only two of the students were not working in computer graphics projects, most of them had no or little experience with 3D mouse (Figure 11). Our population was heterogeneous, consisting of three women and 12 men with ages between 20 and 40, average 25 years old. Each of them tested the three tools and the two devices, performing the 12 tasks.

\subsubsection{Methodology and procedure}

We performed our tests in a Windows XP platform on a standard PC (AGP 4) with a single 3.2 GHz Intel Pentium IV with $512 \mathrm{Mb}$ of RAM, and an ATI Mobility Radeon X600 graphic card with $256 \mathrm{MB}$.

The following steps were applied in the same order to all subjects: 1) instructions about the application and devices were provided; 2 ) a pre-test questionnaire was completed by the subject; 3) unlimited time for training was allowed; 4) the 12 tasks were performed randomly, being the log file recorded; 5) a post-test questionnaire was completed by the subject.
Before being asked to perform the tasks, each user had unlimited time to learn and become familiarized with the application and devices. For this the users had training dataset and tasks (Figure 9d) to practice and only during this stage they could freely switch devices. Once the user felt comfortable, the 12 tasks were performed.

Tasks were selected in random order. After the display of a task specification, the user had to press a start button to actually begin the task. The task did not finish automatically, but when the user felt that he had accomplished it, he/she pressed a button. Then, the new task was randomly chosen by the application and displayed, until all the 12 tasks were accomplished.

Since the hypotheses were based on measuring user's performance during 12 tasks, we designed the experiment with independent and dependent variables as explained below. The log file generated for each user contained the following data items associated with each task: description of the task, total number of grey and red voxels removed from the volume, remaining number of white and red voxels and time spent during the task.

Independent variables are the experiment variables that generate different conditions to be compared. As independent variables we used: age; gender; user's occupation; previous use of 2D and 3D devices; previous use of 2D and 3D applications, like games, CAD, 3D Studio, Blender, etc., and finally the 12 tasks.

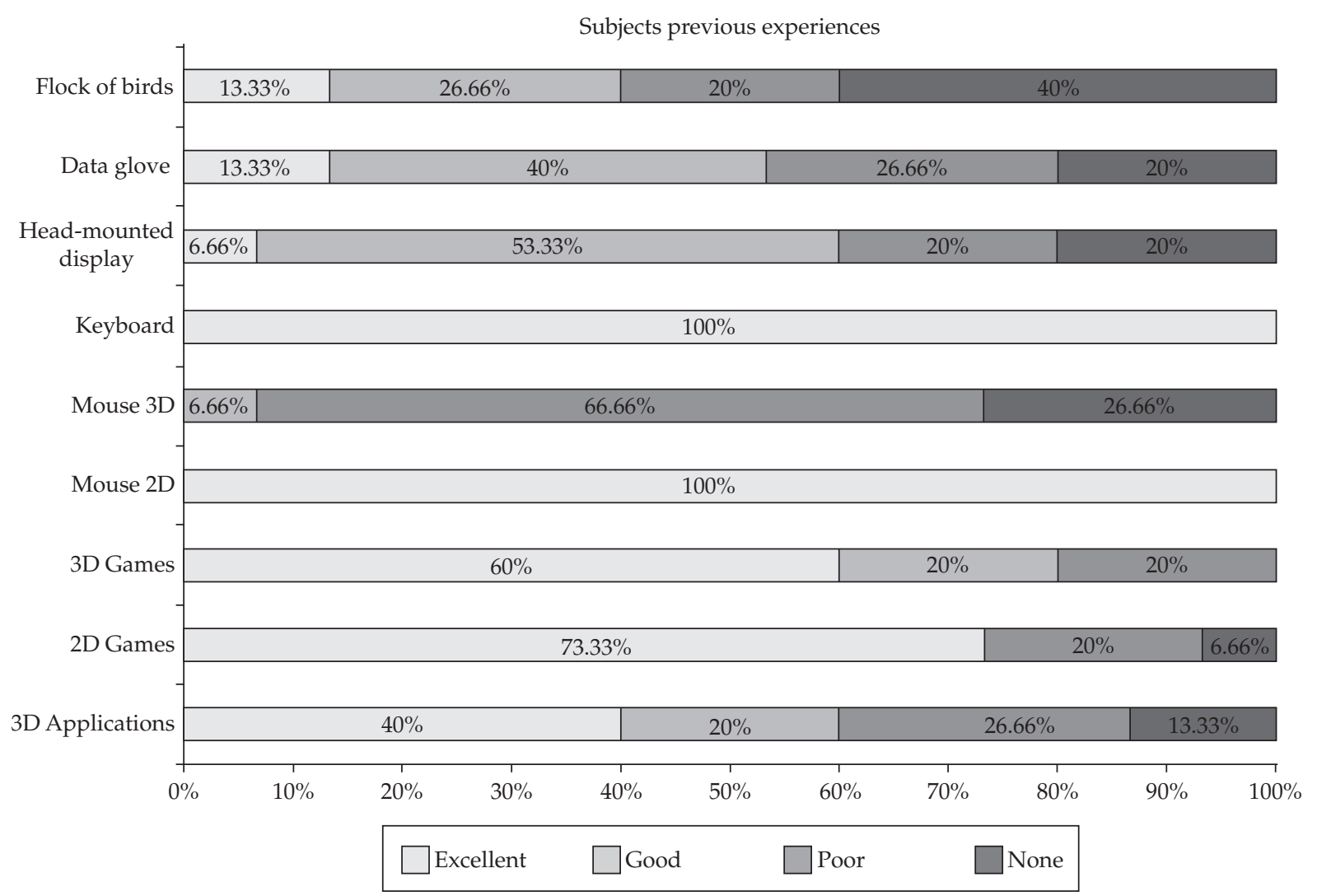

Figure 11. Subjects previous experiences with 2D and 3D applications and devices. 
Dependent variables are measures taken during the execution of tasks. They can be objective, as the time spent to accomplish a task, or subjective, like the level of satisfaction, collected from post-test questionnaires answered by the subjects. The dependent variables used in our experiment were: task completion time; error rate and device preference.

The completion time was measured from the beginning of each task until the user finished it by pressing a button. The error rate (Equation 2) took into account the error after the sculpting task is completed and time spent to perform the task, as given by Equation 2:

$$
\text { error_rate }=\varepsilon * T
$$

where $T$ is completion time and $\varepsilon$ is given by Equation 3:

$$
\varepsilon=\frac{R_{e}+W_{e}}{\# \text { voxels }}
$$

with $R_{\mathrm{e}}$ being the number of remaining red voxels and $W_{\mathrm{e}}$ the number of deleted grey voxels.

Finally, ease of use and device preference were verified through the analysis of questionnaires.

\subsubsection{Results and discussion}

For the statistical analysis of collected data, several variables were considered. These variables were obtained through the pre- and post-test questionnaires and the log files. The pre-test form answered by the subjects provided us with their previous experience with 2D and 3D applications and devices (Figure 11). This table shows that the users had excellent previous experience using the 2D mouse and keyboard, but only a few of them had experience with the 3D mouse.

The correlation between the data from the pre-test questionnaire and the $\log$ files (independent and dependent variables) was calculated.

It was possible to verify that performance with the digger and clipper tasks was correlated to the previous experiences of the subjects with 3D devices, especially when the user had to use the 2D mouse to manipulate the tools and the $3 \mathrm{D}$ mouse to control the volume $(r>0.514, p<0.05)$. Other variables, like age and gender, did not show having influence on performance.

We used ANOVA (Analysis of Variance) to verify the significance of the time and error results obtained from the $\log$ files.

To evaluate the first hypothesis (The manipulation of the tools is faster using a conventional mouse) the time spent by the users to complete each task was analyzed. ANOVA results showed that the difference of the time spent in each task was significant only for the digger $(F=5.308, p<0.02)$ and eraser $(F=4.663, p<0.04)$ tools. When setting the $2 \mathrm{D}$ mouse for controlling volume manipulation, the mean time using the 3D mouse for guiding the tool is higher than the time spent using the 2D mouse for that, both for the digger and the eraser (Figure 12).

In order to verify the second hypothesis (The error rate decreases when users adopt the 3D device to manipulate the tool),

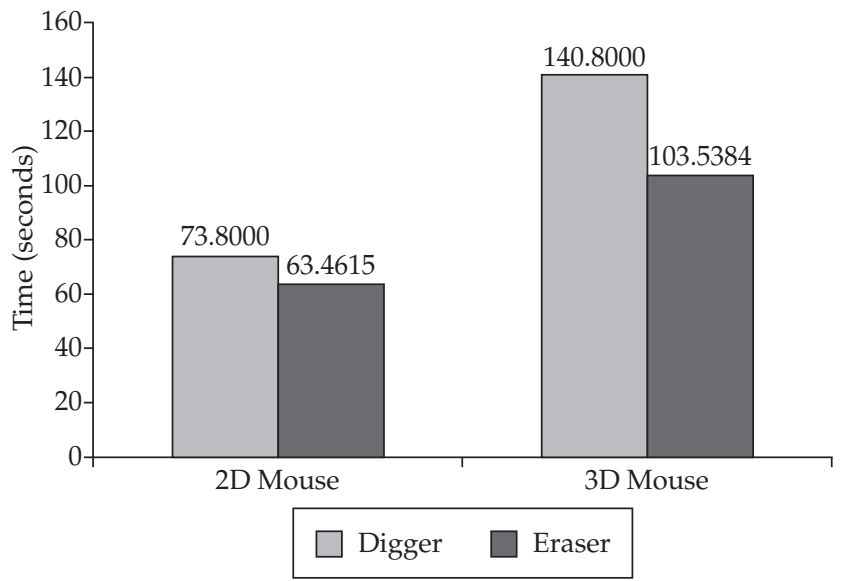

Figure 12. Digger and Eraser mean times (in seconds) when controlling the volume with the 2D mouse.
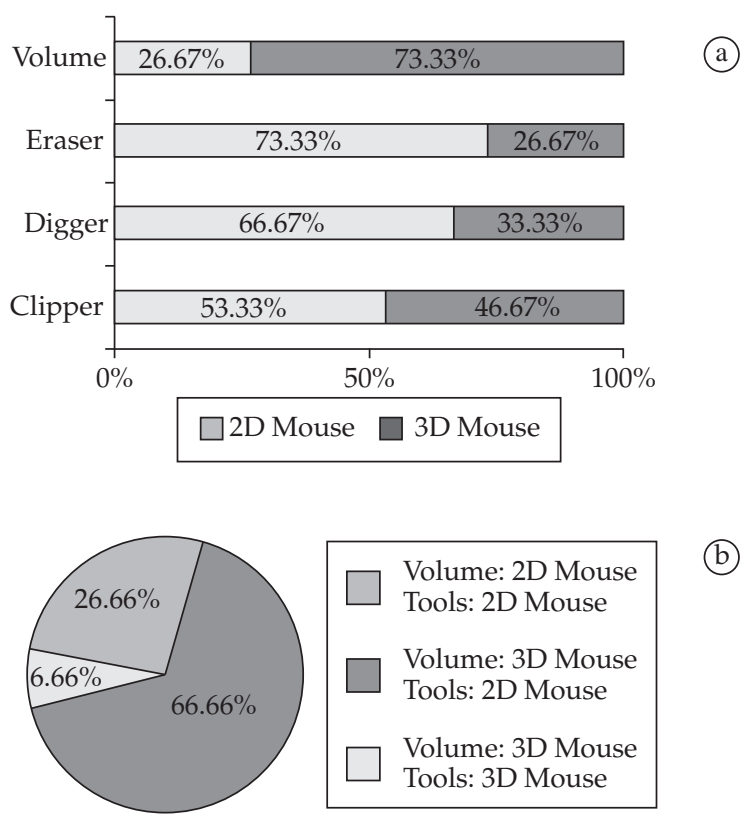

(b)

Figure 13. a) Preferred device to control the volume or tools; and b) preferred combination of devices.

the error rate was calculated multiplying user's error by the time spent in each task, as stated in Equation 2. The user's error was the number of remaining red voxels and deleted grey voxels (recalling that the task was to remove only the red voxels). The difference of performance was not significant according to ANOVA. Therefore the second hypothesis was not confirmed.

The analysis of the post-test questionnaire confirmed the third hypothesis (The users will prefer two-handed input, using both device simultaneously to manipulate volume and tools). Figure 13a shows that the great majority of the users preferred the $2 \mathrm{D}$ mouse to manipulate the tools and the $3 \mathrm{D}$ mouse to control the volume separately. Figure 13b shows that the users also preferred this combination of devices for two-handed input. 


\subsection{Are 3D tools better than $2 D$ ones?}

Regarding the volume sculpting tools, our main goal was to make them intuitive and easy to manipulate. To compare user's performance using 2D and 3D tools we chose the conventional mouse to control both the volume to be sculpted and the actual sculpting tools. This was supported by the results from the first set of experiments. Rotation of the volume was implemented by dragging the mouse with the right button pressed, while the left button is used to apply the tools to the volume.

The manipulation of 2D tools with a conventional mouse is trivial, since all tools have the same degrees of freedom (DOFs), and follows the convention shown in Figure 10. Depth perception was enhanced through visual feedback provided by lighting, fake shadows, guide lines, grid and rulers.

The interface of our application also provides some other features to help with the use of sculpting tools. Axial, coronal and saggital sections of the volume can be shown to allow observation of inner structures of the dataset during the use of digger tools (see Figure 10). The 3D Eraser tool can be rotated and additional translation options are also available.

The evaluation was based on three hypotheses, all of them regarding the use of the two different sets of sculpting tools, i.e., using the virtual pointer metaphor (2D) or the virtual hand (3D):

- H1: Using the 2D volume sculpting tools is faster than using the 3D ones.

- H2: The error rate decreases when using the 3D volume sculpting tools.

- H3: Subjects prefer the 3D volume sculpting tools.

The first hypothesis was conceived upon the fact that regular users are familiar with $2 \mathrm{D}$ interactive tools, and the training period in $3 \mathrm{D}$ tools is not enough to provide them the same skills to manipulate $3 \mathrm{D}$ tools as they would have with $2 \mathrm{D}$ ones. The second hypothesis, on the other hand, was based on the consideration that 3D tasks are better performed using a 3D interaction metaphor, because some problems of mapping from 3D to 2D (as the projection, for example) would be avoided. Finally, the third hypothesis relied on the cognitive benefits from the reduction of the workload of mentally decomposing 3D tasks into unnatural 2D tasks.

\subsubsection{Scenario and subjects}

As before, the experiment consisted on the elimination of red voxels from red-and-grey volumes. In order to facilitate the visualization of cuts and holes, illumination and a marble procedural texture were applied onto the volumes.

The testbed application used 5 phantom datasets as input, each one composed by a stack of images with red and grey pixels: one for training the users before they start the evaluation process (Figure 14a); three for the evaluations of the tools (digger, eraser and clipper), independent of the interaction technique applied (Figures $14 \mathrm{~b}-\mathrm{d}$ ); and a fifth volume to evaluate the use of the three tools concurrently (Figure 14e). All datasets used were opaque. Transparency was used in Figure 14 only to illustrate inner structures.

Tests were performed by 16 Computer Science students, most of them knowledgeable in Computer Graphics. In spite of that, the pre-test questionnaires analysis showed that most of them had little experience with 3D environments. The subjects were between 20 and 29 years old, average of 23.5 and there were only three women in the group. Each of the subjects tested the six sculpting tools in eight tasks.

\subsubsection{Methodology and procedure}

We performed the experiments on a HP Pavillion zd8000 notebook with a single $3.20 \mathrm{GHz}$ Intel Pentium IV CPU, 512 MB of RAM, ATI Radeon X600 PCI Express x16 graphics card with $256 \mathrm{MB}$, running WindowsXP.

The following steps were performed in the same order by each subject: 1) instructions about the application and devices were provided; 2) a pre-test questionnaire was completed; 3) unlimited time for training was allowed; 4) the 8 tasks were performed in a defined order; and 5) a post-test questionnaire was completed.

Tasks were presented to users in a certain order; after the display of a task specification, the user had to press a start button to actually begin the task. The task did not finish automatically, and when the user felt that he/she had accomplished it, he/she pressed a button. Then, the new task was displayed by the application, until all the 8 tasks were accomplished.

Users were separated into two groups with the same size to avoid the order factor: one group tested the 2D set of tools first than the 3D, while the other did the opposite. Each set of tools was evaluated in the same order: the digger tool was followed by the eraser and clipper, and the task to evaluate all
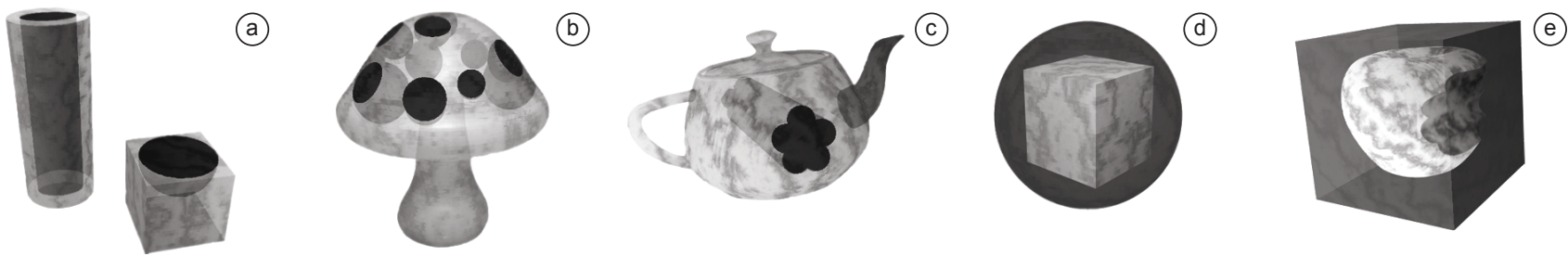

Figure 14. Datasets for experiment 2: a) a cube and a cylinder, for training; b) a mushroom, for the digger evaluation; c) a teapot, for the eraser evaluation; d) a cube, for the clipper evaluation; and e) a bitten apple, for the evaluation of the three tools at the same time. 
the tools together was the last one. This order was selected based on results obtained from the previous experiment.

For each user, a log file was generated with the following data items associated with each task: task id; total number of grey and red voxels of the volume; remaining number of grey and red voxels; time spent in the task; and history of actions performed.

As independent variables we used: age; gender; user's occupation; previous use of 2D and 3D devices; previous use of 2D and 3D applications such as image editors, games, CAD, 3D Studio Max, Blender, etc.; and the eighth tasks.

The dependent variables considered were: task completion time; error rate; and device preference. The completion time was measured from the beginning of each task until the user finished it by clicking on a button in the system interface. The error rate is calculated as in the first experiments (Equation 2).

Ease of use and device preference were verified through the analysis of questionnaires.

\subsubsection{Results and discussion}

The pre-test form answered by the subjects provided us with their previous experience with 2D and 3D applications. The correlation between the data from the pre-test questionnaires and the data saved in the log files was calculated. There was no correlation between previous experiences that the subjects had with the performance obtained during this second set of tests. Other variables like age and gender did not show any correlation too.

As before, we used ANOVA to verify the significance of time and error results obtained from log files. To evaluate the first hypothesis (Using the 2D volume sculpting tools is faster than using the $3 D$ ones) the time spent by the users to complete each task was analyzed.

ANOVA test results showed that the difference in the time spent in each task was significant only for tasks where we allowed the use of all the tools together $(F=9.433$, $p<0.00494)$. In this case, the first hypothesis was confirmed, because the mean time of sculpting the volume using the 3D tools was much higher than the mean time spent with the 2D tools (Figure 15).

In order to verify the second hypothesis (The error rate decreases when using the 3D volume sculpting tools), the error rate was calculated multiplying the user's error by the time spent in each task, as stated in Equation 2. The difference of performance was significant according to ANOVA when the users tested the eraser $(F=42.222, p<6.90207 \mathrm{E}-07)$ and the clipper tools $(F=4.264, p=0.0490)$. In these cases, the second hypothesis was confirmed because the error rate when using the 2D tools was higher than when using the $3 \mathrm{D}$ ones (Figure 16).

The analysis of post-test questionnaires confirmed the third hypothesis (Subjects prefer the 3D volume sculpting tools). Figure 17 shows that most of the users preferred 3D interaction techniques for sculpting volumes. The only case where this hypothesis was not confirmed was for eraser tools

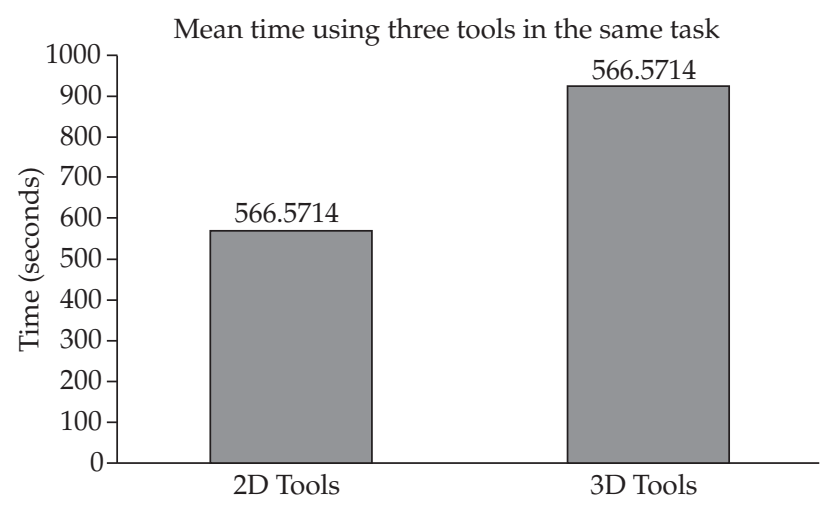

Figure 15. Mean time for tasks combining the use of eraser, digger and clipper concurrently.

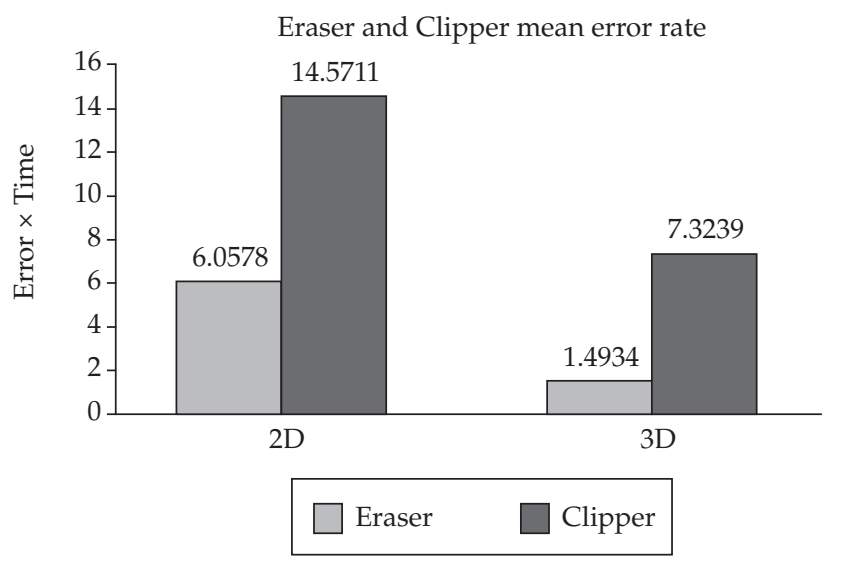

Figure 16. Mean error rate for eraser and clipper tools.

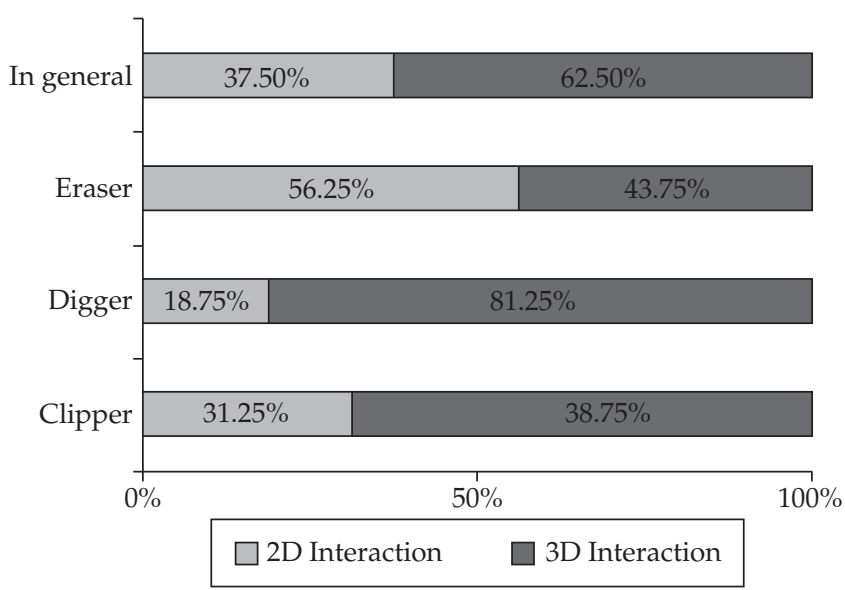

Figure 17. Preference of the users after the evaluation of $2 \mathrm{D}$ and $3 \mathrm{D}$ volume sculpting tools.

evaluation. Even though the users preferred the 2D Eraser, the performance analysis (Figure 16) shows that the 2D Eraser was much worse than the $3 D$ Eraser. This is probably due to the illusion that the $2 D$ Eraser removes the voxels as if it was a cylinder perpendicular to the view plane, instead of a cone. The illusion is caused by the perspective projection intrinsic to the Aperture Selection technique. 


\section{Filling Tools}

Volumetric datasets can also be used for modelling missing structures, as exemplified in Figure 2. Such structures can be sculpted by inserting material in selected voxels, and can be used to build models of prosthesis based on isosurfaces extracted from the sculpted volumes.

Since our volume sculpting tools were developed based on selection and marking of voxels inside volumetric datasets, it was a straightforward idea to devise tools that instead of cutting out volume parts would add material to the selected voxels. We developed two such filling tools as a proof-of-concept of our idea. A mirror and a filler tool were implemented based on the $3 \mathrm{D}$ virtual hand interaction metaphor already employed in our 3D Clipper and Digger tools (Figure 18).

The following subsections describe these two filling tools and Section 7 reports their assessment in a practical application.

\subsection{Mirror}

This tool was designed based on the existing symmetry of many structures of the human body (like the head, for example). A reflection plane, dividing the volume in two parts, acts by copying the content of one side to the other. The position and orientation of the reflection plane are interactively selected by the user, the direction of mirroring represented by an arrow on the plane representation (Figure 18a). The direction of mirroring represents the part of the volume that must be the target (selected) area for copying purposes, while the opposite direction corresponds to the source region.

The mirroring process works by calculating for each voxel $V$ of the selected part of the volume, the coordinates of $V^{\prime}$, reflected by the plane (Figure 19a). $V^{\prime}$ coordinates are then used to access the voxel in the source region for the copying operation. If there exists visible material on $V^{\prime}, V$ will be set
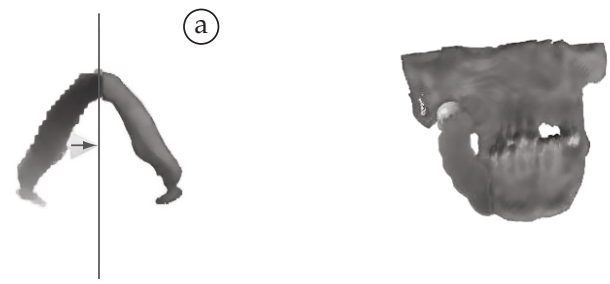

(b)

Figure 18. Filling tools: a) Mirror, and b) Filler.
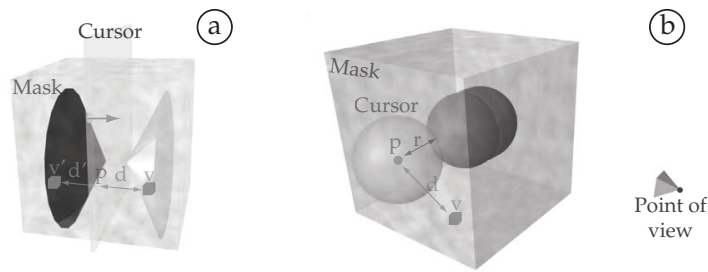

Figure 19. Implementation of filling tools: a) Mirror, b) Filler. to the same value, representing the insertion of the same material in $V$. Assuming $d$ the distance between $V$ and its orthogonal projection $P$ on the reflection plane: the mirrored voxel $V^{\prime}$ is located at the absolute distance $a b s(d)$ of $P$, on the same direction of the vector $v(V-p)$.

\subsection{Filler}

This tool was created for providing direct insertion of material in voxels at any position in the volume (Figure 18b). The 3D cursor can be moved, and works exactly as the 3D Digger tool, being a sphere of radius $r$ and center $P$ specified by the user. By moving the sphere in the volume space, each voxel visited by the tool is marked with inserted material if the distance $d$ of the voxel center to $P$ is smaller than $r$ (Figure 19b). It should be emphasized that the Filler does not overwrite existing material, by first checking the current value of the voxels inside the sphere. The voxels are filled only if they have background values.

\subsection{Implementation of filling tools}

Mirror and filler tools were integrated in the same texturebased volume rendering system presented in Figure 4 . The same texture used as a mask representing the marked information to be excluded from the rendering by the volume sculpting tools is used for representing the inserted material. Actually the filling tools use a different color channel (B), while the clipping tools use the A values for visibility information. This way the user can freely add and remove voxels before concluding the sculpting process since she/he manipulates only the 3D mask.

Following the same strategy of the volume clipping tools, the filling tools were implemented by specific shaders.

The shader FSMirror (Figure 20) is applied for each fragment of the proxy geometry, and the code shows the implementation considering a plane that moves along the $X$ axis. The Filler tool is implemented by the fragment shader shown in Figure 21.

The integration of the filling tools to the pipeline shown in Figure 3 required a modification in the following way: in the update part, the (SculptMap) now represents two masks with the visibility information and the inserted material, which are updated by all the tools; the drawing part (and consequently the shader FSCombiner) now combines the content of both masks with the original dataset. The modified FSCombiner is shown in Figure 22.

\section{Evaluation of the Filling Tools}

Since the previous experiments showed that $3 \mathrm{D}$ volume sculpting tools were the preferred ones by the users while yielding lower error rate, we planned the assessment of the filling tools as a scenario-based evaluation approach, i.e., by describing a practical usage scenario.

In order to assess the effectiveness and quality (efficiency, precision, intuitiveness and comfort) of the filling tools, we 


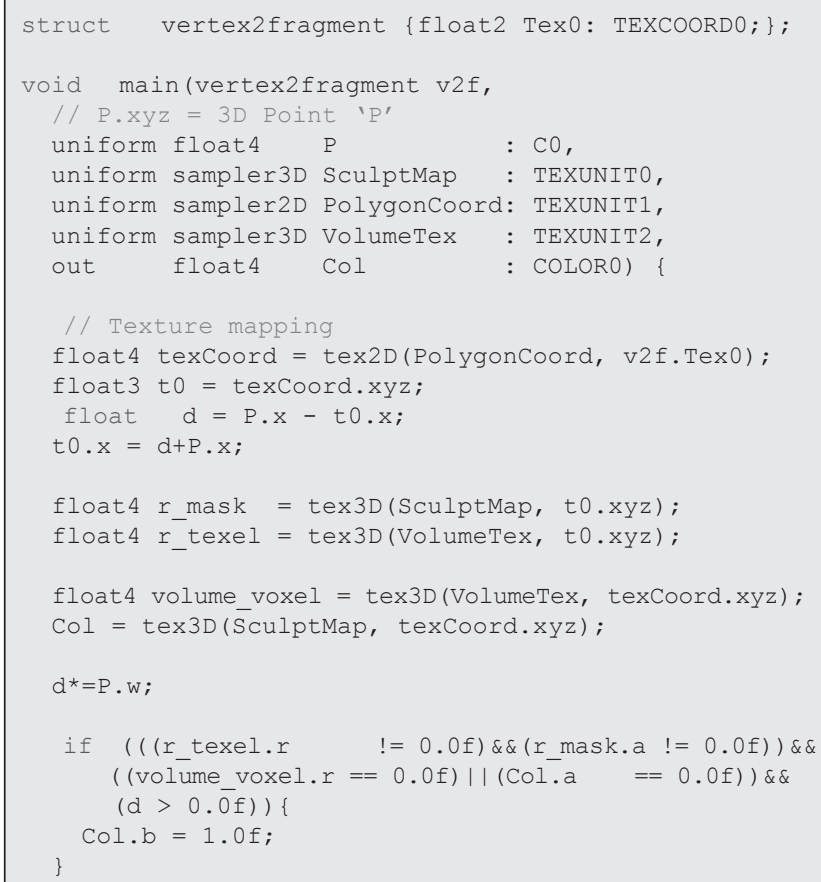

Figure 20. Cg code for FSMirror.

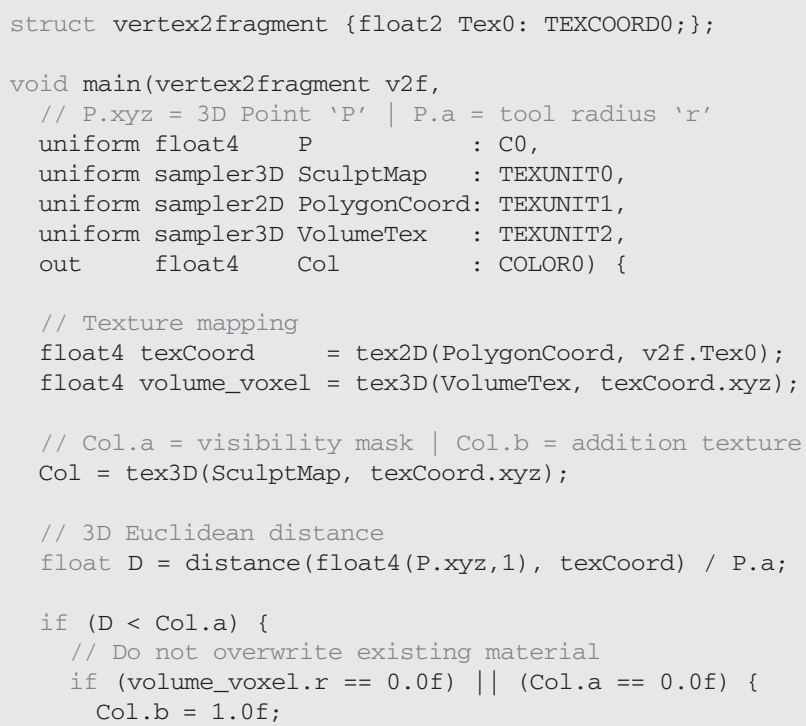

Figure 21. Cg code for FSFiller.

describe how these tools could be used by a biomodel designer on a dataset of a cranium from an injured patient, which has lost part of the jaw as result of a tumor (Figure 2a). The jaw is partially shifted, which can be seen by the positions of the incisor teeth, probably due to the lack of structural support.

Since the maxilla is similar in both sides of the cranium, our biomodel designer starts using the mirror tool (Figure 23, top left). She moves the reflection plane representation using the conventional mouse until the desired position and

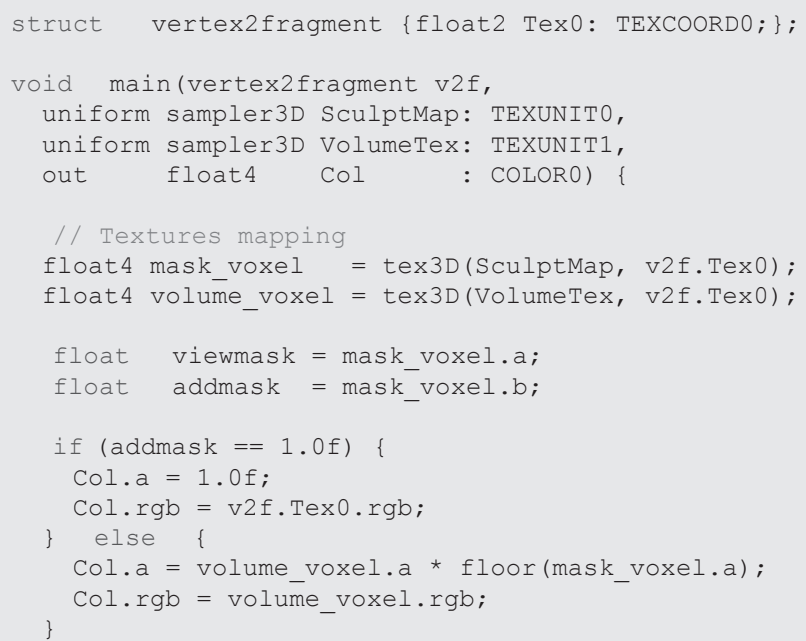

Figure 22. $\mathrm{Cg}$ code for FSCombiner with the additive mask.
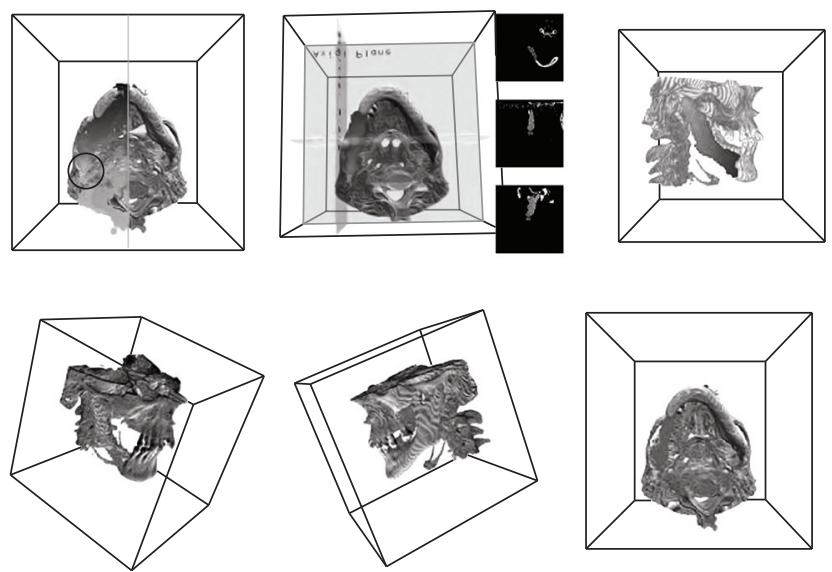

Figure 23. Case study of filling tools. Top images, left to right: modeling a prosthesis for an injured jaw. After reflecting one of the sides by applying the mirror tool, the resulting material was segmented using clipping tools. The application of filler tool completes the modeling process: three additional windows show axial, coronal and sagittal slices at the cursor position. Bottom row: the resulting volume is presented in the three final images.

orientation are achieved, and apply the mirroring operation. However, due to the fact that the jaw is shifted and that usually it presents some asymmetry, the simple application of this tool does not correctly rebuild the new structure. More material than needed is added to that part of the volume as well as there are voxels that still need to be filled in. So, the designer chooses to inspect the condyle (see circled region in Figure 23, top center) with axial, coronal and sagittal views. To do that she moves the cursor and use the views to reach the new desired position. Then, in successive steps, she uses the clipping tools and filler to refine the added material, reaching the result shown in Figure 23, top right. At this point, the resulting rendered volume shows the original voxels as well as the added material. 
As a final step, the designer can submit the combined volume to a mesh extraction method and obtain a geometric model of the added structure. This model could then be used with rapid prototyping tools to build physical models to serve as implants for our patient.

\section{Final Remarks}

We presented a set of volume sculpting tools using both 2D and 3D interaction techniques to allow exposing inner parts of volumes. We also investigated filling tools to allow the user to build structures that fit in the volume data, filling gaps for building prosthesis, for example. The proposed tools are based on the multi-texturing facilities provided by modern GPUs and implemented in special fragment shaders that discard fragments based on visibility information. This way we achieved real-time performance in volume and tools manipulation.

A first set of experiments with users allowed us to evaluate the use of different devices to interact with the application for manipulating the sculpting tools and controlling the volume position and orientation. Analysis of results showed that, in the few cases where the difference of performance was significant, using the 2D mouse to control the tools allowed a better time performance. Moreover, although the results were not significant under ANOVA, the error rate was lower when using the 3D mouse for controlling the tools. Analysis of the questionnaires showed that the users prefer to use both hands and both devices (2D mouse for moving the tool and 3D mouse for manipulating the volume) to perform the sculpting tasks. As a conclusion, collected data shows that better results are obtained when the mapping between the interaction in the real and virtual worlds is direct.

A second set of experiments was carried out to find the best set of tools (2D or 3D) from the user's point of view. Analyses of results showed that when all tools that are based on the same interaction metaphor are used together, the mean time of those that use 3D interaction is higher. This would be interpreted as a disadvantage of our 3D volume sculpting tools if we had taken only time as a performance measure. Significant results favoring the 3D tools were obtained when analyzing the error rate. Users had fewer errors when using techniques based on the virtual hand metaphor. The preference of the users, obtained from questionnaires, was also for the use of the 3D volume sculpting tools.

Based on results favoring 3D interaction tools we developed filling tools so the user can build structures that can be further visualized or extracted as meshes. These tools have direct application to the design of biomedical models to serve as prosthesis and implants. Two case studies were conducted as an assessment of such tools, one was reported here. Next steps of our work are concentrated on validating filling tools by building a physical prototype of the added structure in order to compare it with the prosthesis built by designers following conventional procedures.

\section{Acknowledgements}

We gratefully acknowledge the experimental users as well as all colleagues from the Computer Graphics Lab at UFRGS for suggestions and helpful comments. We also acknowledge the financial support from $\mathrm{CNPq}$, and the Design and Material Selection Laboratory (LdSM) from UFRGS, mainly Liciane Bertol, for sharing with us her experience in biomodel design and providing us the data we used in the case studies

\section{References}

1. Bowman DA and Hodges LF. An Evaluation of Techniques for Grabbing and Manipulating Remote Objects in Immersive Virtual Environments. Providence, RI, USA: ACM; 1997. p. 35-38.

2. Bowman DA, Johnson D and Hodges LF. Testbed Evaluation of Virtual Environment Interaction Techniques. Presence: Teleoperators and Virtual Environments 2001;10(1):75-95.

3. Bruckner S and Gröller E. Enhancing Depth-Perception with Flexible Volumetric Halos. IEEE Transactions on Visualization and Computer Graphics 2007;13(6):1344-1351.

4. Bruckner S and Gröller ME. VolumeShop: An Interactive System for Direct Volume Illustration. Proceedings of the IEEE Conference on Visualization; 2005. p. 671-678.

5. Buxton W and Meyers BA. A Study in Two-Handed Input. Proceedings of the SIGCHI Conference on Human Factors in Computing Systems; 1986; Boston, MA, USA. p. 321-326.

6. Chen HJ, Samavati FF and Sousa MC. GPU-based point radiation for interactive volume sculpting and segmentation. The Visual Computer 2008;24(7):689-698.

7. Correa CD and Silver D. Programmable shaders for deformation rendering. GH '07: Proceedings of the 2007 ACM SIGGRAPH/EUROGRAPHICS conference on Graphics hardware; 2007; Aire-la-Ville, Switzerland. p. 89-96.

8. Pinto FM and Dal Sasso Freitas CM. Design of Multidimensional Transfer Functions Using Dimensional Reduction. EuroVis07: Joint Eurographics - IEEE VGTC Symposium on Visualization; 2007; Sweden. p. 131-138.

9. Diepstraten J, Weiskopf D and Ertl T. Transparency in Interactive Technical Illustrations. Computer Graphics Forum 2002;21(3):125-148.

10. Ebert D and Rheingans P. Volume Illustration: Non-Photorealistic Rendering of Volume Models. Proceedings of the IEEE Conference on Visualization; 2000. p. 195-202.

11. Engel K, Kraus M and Ertl T. High-Quality Pre-Integrated Volume Rendering Using Hardware-Accelerated Pixel Shading.Proceedings of the ACMSIGGRAPH/EUROGRAPHICS Workshop on Graphics Hardware, HWWS; 2001; Los Angeles, CA, USA. p. 9-16

12. Forsberg A, Herndon $K$ and Zeleznik R. Aperture Based Selection for Immersive Virtual Environments. Proceedings of the ACM Symposium on User Interface Software and Technology, UIST; 1996; Seattle, WA, USA. p. 95-96.

13. Galyean TA and Hughes JF. Sculpting: an interactive volumetric modeling technique. Proceedings of the Annual Conference on Computer Graphics and Interactive Techniques, SIGGRAPH, 18; 1991; Las Vegas, NV, USA. p. 267-274. 
14. Van Gelder A and Kim K. Direct Volume Rendering with Shading via Three-Dimensional Textures. Proceedings of the Symposium on Volume Visualization, VVS; 1996; San Francisco, CA, USA. p. 23-30.

15. Hinckley K, Pausch R, Goble JC and Kassell NF. A Survey of Design Issues in Spatial Input. Proceedings of the Annual ACM SIGGRAPH Symposium on User Interface Software and Technology, UIST, 7; 1994; Marina del Rey, CA, USA. p. 213-222.

16. Huff R, Dietrich CA, Nedel LP, Dal Sasso Freitas CM, Dihl Comba JL and Olabarriaga SD. Erasing, Digging and Clipping in Volumetric Datasets with One or Two Hands. Proceedings of the ACM International Conference on Virtual Reality Continuum and Its Applications, VRCIA; 2006; Hong Kong, 2006. p. 271-278.

17. Islam S, Silver D and Chen M. Volume Splitting and Its Applications. IEEE Transactions on Visualization and Computer Graphics 2007;13(2):193-203.

18. Kindlmann $G$, Whitaker $R$, Tasdizen $T$ and Möller $T$. Curvature-Based Transfer Functions for Direct Volume Rendering: Methods and Applications. Proceedings of the IEEE Conference on Visualization, VIS; 2003. p. 513-520.

19. Kniss J, Kindlmann G and Hansen C. Interactive Volume Rendering Using Multi-Dimensional Transfer Functions and Direct Manipulation Widgets. Proceedings of the IEEE Conference on Visualization, VIS ; 2001; San Diego, CA, USA. Washington: IEEE Computer Society. p. 255-262

20. Leganchuk A, Zhai S and Buxton W. Manual and Cognitive Benefits of Two-Handed Input: An Experimental Study. ACM Transactions on Computer-Human Interaction 1998; 5(4):326-359.

21. Liang J and Green M. Geometric modeling using six degrees of freedom input devices. Proceedings of the International Conference on Computer Aided Design and Computer Graphics, CAD/CG, 3; 1993; Beijing, China. p. 217-222.

22. Lorensen WE. Geometric clipping using boolean textures. Proceedings of the IEEE Conference on Visualization; 1993; San Jose, CA, USA. p. 268-274.

23. McGuffin M, Tancau L and Balakrishnan R. Using deformations for browsing volumetric data. Proceedings of the IEEE Conference on Visualization, VIS; 2003. p. 401-408.

24. Mine MR. Virtual Environment Interaction Techniques. Chapel Hill, NC, USA: University of North Carolina at Chapel Hill, Computer Science Department; 1995. Technical report

25. Mine MR, Brooks Jr. FP and Sequin CH. Moving objects in space: exploiting proprioception in virtual-environment interaction. Proceedings of the Annual Conference on Computer Graphics and Interactive Techniques, SIGGRAPH, 24; 1997; Los Angeles, CA, USA. p. 19-26

26. Nedel LP, Dal Sasso Freitas CM, Jacob LJ and Pimenta MS. Testing the Use of Egocentric Interactive Techniques in Immersive Virtual Environments. Proceedings of IFIP TC13 International Conference on Human-Computer Interaction, INTERACT, 9; 2003; Zürich, Switzerland. Amsterdam: IOS Press. p. 471-478.

27. Parviainen J, Sainio N and Raisamo R. Perceiving Tools in 3D Sculpting. Proceedings of the Asia-Pacific Computer and Human
Interaction, APCHI, 6; 2004; Rotorua, New Zealand. Berlin: Springer-Verlag. p. 328-337.

28. Pfister H, Hardenbergh J, Knittel J, Lauer H and Seiler L. The VolumePro Real-Time Ray-Casting System. Proceedings of the Annual Conference on Computer Graphics and Interactive Techniques, SIGGRAPH, 26; 1999; Los Angeles, CA, USA. p. 251-260.

29. Pfister H, Lorensen WE, Schroeder WJ, Bajaj CL and Kindlmann GL. The transfer function bake-off (panel session). Proceedings of the IEEE Conference on Visualization, VIS; 2000. p. 523-526.

30. Pflesser B, Petersik A, Tiede U, Höhne KH and Leuwer R. Volume cutting for virtual petrous bone surgery. Computer Aided Surgery 2002;7(2):74-83.

31. Pierce JS, Forsberg AS, Conway MJ, Hong S, Zeleznik RC and Mine MR. Image Plane Interaction Techniques in 3D Immersive Environments. Providence, RI, USA: ACM; 1997. P. 39-43.

32. Poupyrev I, Billinghurst $M$, Weghorst $S$ and Ichikawa T. The Go-Go Interaction Technique: Non-Linear Mapping for Direct Manipulation in VR. Proceedings of the Annual ACM SIGGRAPH Symposium on User Interface Software and Technology, UIST, 9; 1996; Seattle, WA, United States. p. 79-80

33. Poupyrev I, Weghorst S, Billinghurst M and Ichikawa T. Egocentric Object Manipulation in Virtual Environments: Evaluation of Interaction Techniques. Computer Graphics Forum 1998; 17(3):41-52.

34. Rezk-Salama C, Engel K, Bauer M, Greiner G and Ertl T. Interactive Volume Rendering on Standard PC Graphics Hardware Using Multi-Textures and MultiStage Rasterization. Proceedings of the ACM SIGGRAPH/ EUROGRAPHICS Workshop on Graphics Hardware, HWWS; 2000; Interlaken, Switzerland. p. 109-118.

35. Sachs E, Roberts A and Stoops D. 3-Draw: A Tool for Designing 3D Shapes. IEEE Computer Graphics and Applications 1991; 11(6):18-26.

36. Song D and Norman M. Nonlinear Interactive Motion Control Techniques for Virtual Space Navigation. Proceedings of IEEE Virtual Reality Annual International Symposium, VR; 1993; Seattle, WA, USA. p. 111-117.

37. Wang SW and Kaufman AE. Volume Sculpting. Monterey, CA, USA: ACM; 1995. p. 151-156.

38. Weiskopf D, Engel $\mathrm{K}$ and Ertl T. Interactive Clipping Techniques for Texture-Based Volume Visualization and Volume Shading. IEEE Transactions on Visualization and Computer Graphics 2003; 9(3):298-312.

39. Weiskopf D, Engel $K$ and Ertl T. Volume Clipping via Per-Fragment Operations in Texture-Based Volume Visualization. Proceedings of the IEEE Conference on Visualization, VIS; 2002; Boston, MA. p. 93-100.

40. Westermann R and Ertl T. Efficiently Using Graphics Hardware in Volume Rendering Applications. Proceedings of the Annual Conference on Computer Graphics and Interactive Techniques, SIGGRAPH, 25; 1998; Orlando, FL, USA. p. 169-177.

41. Wu Y and Qu H. Interactive Transfer Function Design Based on Editing Direct Volume Rendered Images. IEEE Transactions on Visualization and Computer Graphics 2007; 13(5):1027-1046. 


\section{Color Plate}
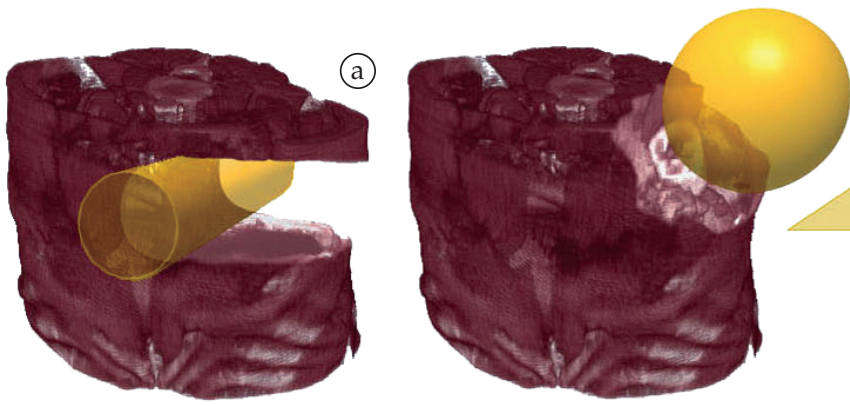

(b)

(c)
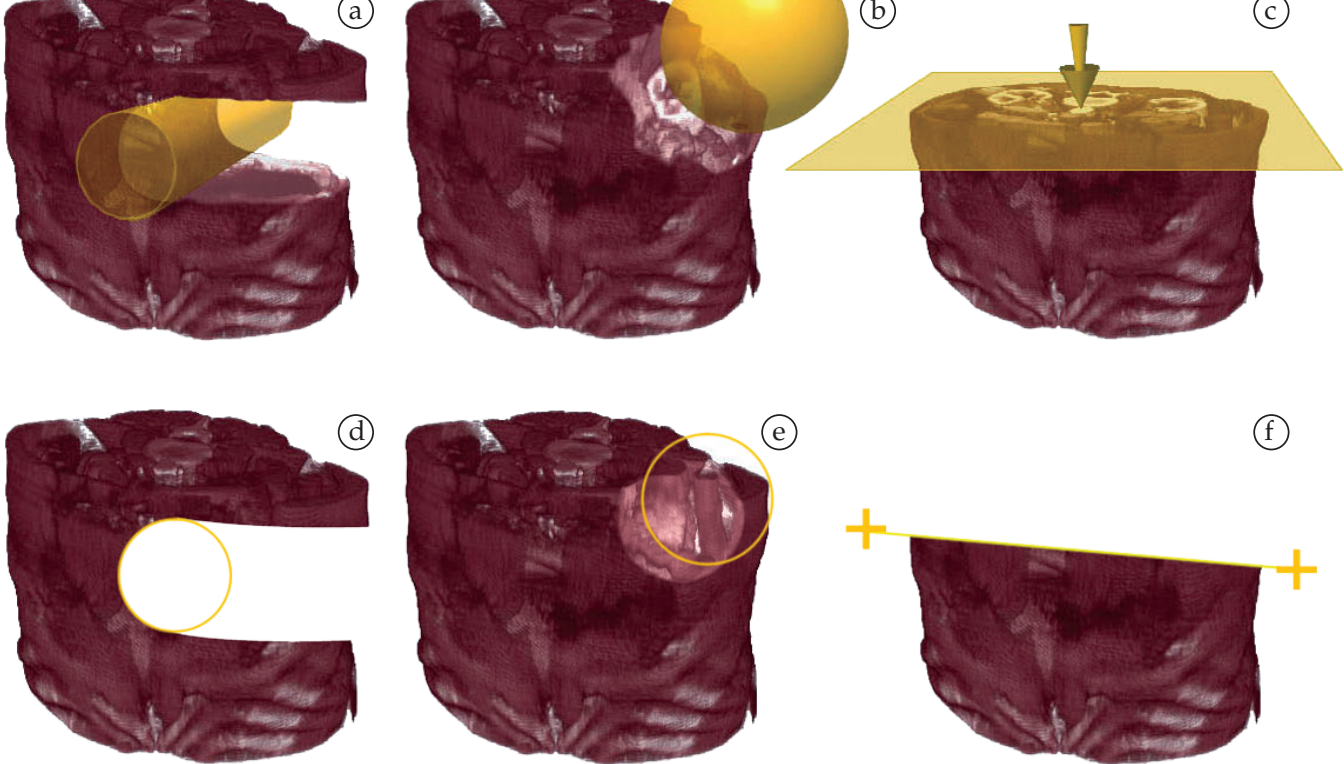

(†)

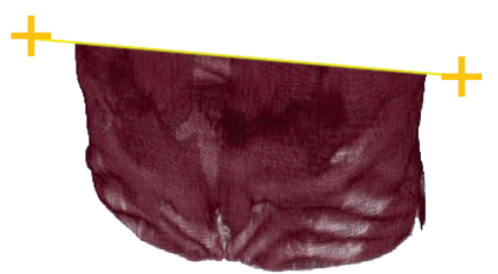

Figure 1. Volumetric Sculpting Tools: a) 3D Eraser, b) 3D Digger, c) 3D Clipper, d) 2D Eraser, e) 2D Digger and f) 2D Clipper.
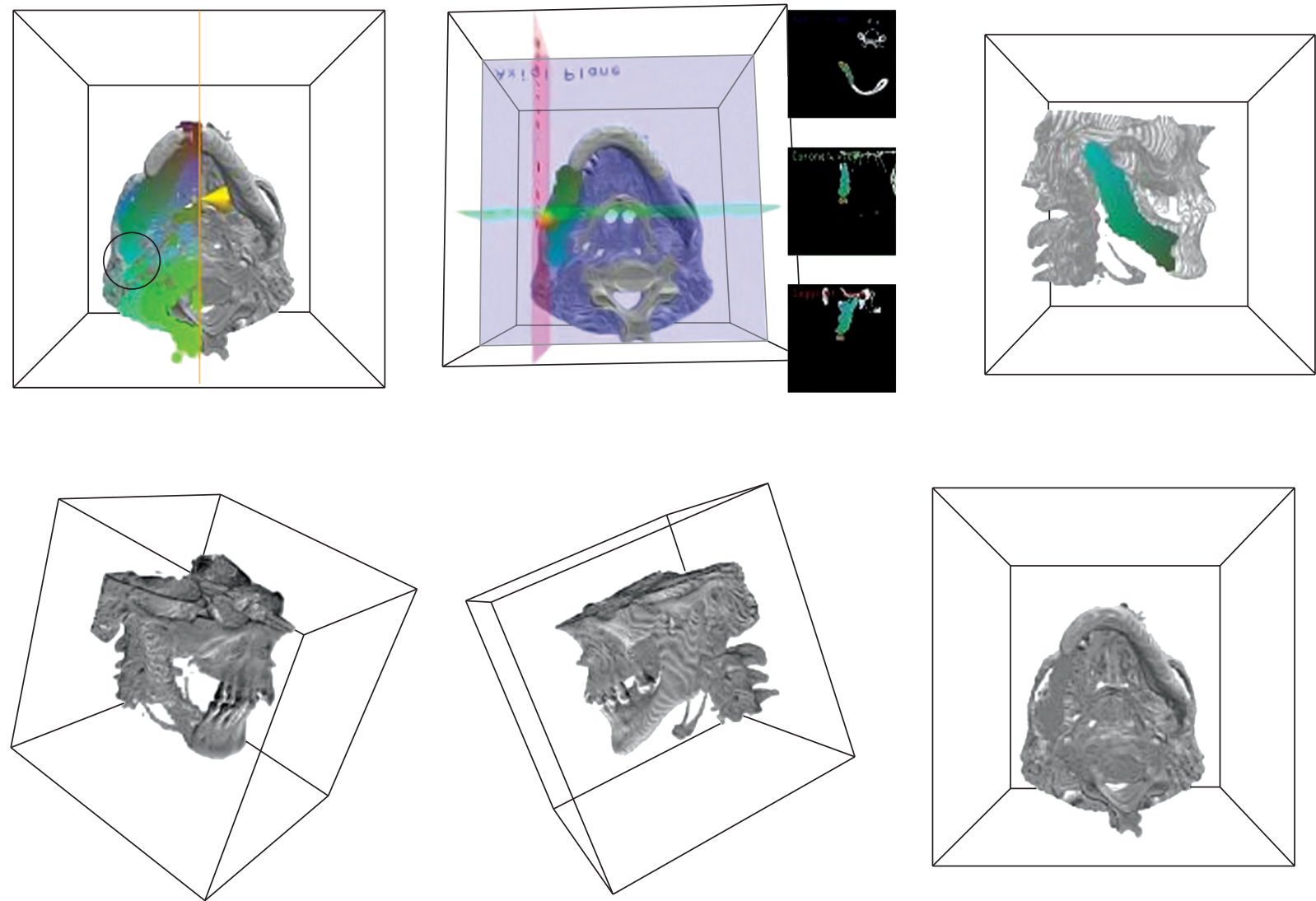

Figure 23. Case study of filling tools. Top images, left to right: modeling a prosthesis for an injured jaw. After reflecting one of the sides by applying the mirror tool, the resulting material was segmented using clipping tools. The application of filler tool completes the modeling process: three additional windows show axial, coronal and sagittal slices at the cursor position. Bottom row: the resulting volume is presented in the three final images. 